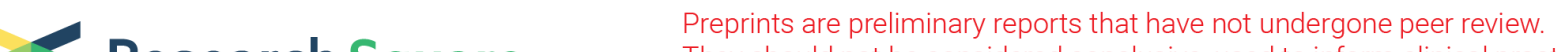 Research Square
or referenced by the media as validated information.
}

\section{RinasciMENTE. An Internet-Based Self-Help Intervention to Cope With Psychological Distress Due to COVID-19: A Study Protocol for a Randomized Controlled Trial}

\author{
Vanessa Bertuzzi \\ 8541-5357 \\ Michelle Semonella \\ Bar-llan University \\ Gerhard Andersson \\ Linköping University: Linkopings universitet \\ Gian Mauro Manzoni \\ eCampus University: Universita degli Studi eCampus \\ Gianluca Castelnuovo \\ Università Cattolica del Sacro Cuore: Universita Cattolica del Sacro Cuore

\section{Enrico Molinari} \\ Università Cattolica del Sacro Cuore: Universita Cattolica del Sacro Cuore \\ Giada Pietrabissa ( $\nabla$ giada.pietrabissa@unicatt.it) \\ Università Cattolica del Sacro Cuore: Universita Cattolica del Sacro Cuore
}

Università Cattolica del Sacro Cuore: Universita Cattolica del Sacro Cuore https://orcid.org/0000-0002-

\section{Research Article}

Keywords: COVID-19, Internet-based intervention, Self-help, Cognitive-behavioral therapy, Psychological distress, Randomized controlled trial, Clinical psychology

Posted Date: March 8th, 2022

DOI: https://doi.org/10.21203/rs.3.rs-1372315/v1

License: (1) This work is licensed under a Creative Commons Attribution 4.0 International License. Read Full License 


\section{Abstract}

Background This study aims to evaluate the feasibility and effectiveness of the RinasciMENTE program, an Internet-based self-help intervention based on Cognitive Behavioral Therapy (CBT) principles and techniques in supporting individuals experiencing psychological impairments during the COVID-19 pandemic. A randomized controlled trial (RCT) design with random allocation at the level of individual will be conducted to compare the impact of the RinasciMENTE program with a waiting list control in improving the psychological functioning of the general population during the COVID-19 pandemic.

Methods A minimum sample of 128 participants experiencing mild/subthreshold levels of psychological symptoms during the COVID-19 pandemic will be recruited. After the initial screening, participants will be randomly assigned to either the experimental group or the control condition. The program will last 2 months, during which participants will receive 8 weekly CBT treatment modules. The impact of the RinasciMENTE program on selected primary and secondary psychological outcomes will be tested at the end of the intervention (2 months), 6- and 12-month follow-up.

Discussion We expect people to show an increased level of psychological functioning, and to acquire the skills and self-confidence necessary to deal with the psychological consequences of the COVID-19 outbreak and its related social isolation during and following the pandemic.

Trial registration ClinicalTrials.gov NCT0497903 Registered on 28 May 2021.

\section{Background}

In the hope of reducing the transmission of Coronavirus Disease 2019 (COVID-19) governments around the world have implemented unprecedentedly strict preventive measures, such as home confinement, mobility restrictions, and social distancing $(1,2)$. However, the COVID-19 pandemic and the resulting economic recession (3) have negatively affected the mental health of many people (4). The most common mental disorders emerging were anxiety and depression, obsessive-compulsive symptoms, insomnia, and post-traumatic stress disorder symptoms (5-9). These were not only a direct consequence of the pandemic but also largely driven by the effects of prolonged social isolation. Although necessary to limit the spread of the epidemic, social isolation resulted in increased sedentary lifestyle (2) and other COVID-19-related stressors including fears of infection, frustration and boredom, and lack of in-person contacts $(10,11)$. Italy was one of the most affected countries during the outbreak, initially accounting for over 223.000 infected individuals and more than 31.000 deaths (12). In May 2021, 4.111.110 people resulted having contracted the virus, and 122.833 of them died as a consequence $(13,14)$.

In this scenario, it is crucial to assess the level of psychological distress and to provide ad hoc psychological interventions to support the individuals' wellbeing in both the general population $(15,16)$ and at-risk groups $(17,18)$ during the COVID-19 pandemic. 
Accordingly, evidence from previous epidemics highlights the risk for long-term mental health issues (19) and emphasizes the need for continued support during and after the pandemic (20).

Many health organizations have already committed resources to support the mental well-being of the individuals, adapting existing standard programs to meet evolving demands caused by COVID-19 (21). In this regard, cognitive-behavioral techniques including restructuring of cognitive bias, as well as activity planning and relaxation, have been shown particularly useful in addressing psychological distress (2225).

Cognitive-behavioral therapy (CBT) is a time-sensitive, structured, present-oriented psychotherapy aimed at helping people identify and change thinking and behavior patterns that are harmful or ineffective, replacing them with more accurate thoughts and functional behaviors.

Still, given that face-to-face treatment is not always viable in these circumstances (26) a new way to deliver psychological treatments is urgently required, and a potentially practical solution is to deliver therapy remotely $(14,27-30)$.

Many systematic reviews and randomized controlled trials (RCTs) demonstrated the utility and the efficacy of Internet-based interventions in supporting people experiencing psychological problems (3133).

Advantages include improved access for individuals (26) who have variable schedules, an important workload, or who are afraid of being contaminated through face-to-face contacts (34-36), as well as cost-effectiveness compared to face-to-face treatment.

Due to its features, CBT well-suits many aspects of distance therapy. First, it is a talking therapy, and this aspect can be relatively easily retained remotely. It also emphasizes the importance of the person making changes and working on specific tasks between sessions to bring about the change (37); this is perfectly consistent with working remotely. Moreover, with distance therapy, the patient may be less likely to attribute the progress to the therapist and more likely to have an improved sense of self-efficacy (38). One of the goals of CBT is to "become your therapist" by learning skills people can use on their own after treatment to keep feeling well.

Research shows that CBT delivered digitally (iCBT) is effective in reducing symptoms of social anxiety disorder $(39,40)$, generalized anxiety disorder $(41,42)$, panic disorder $(43)$, major depressive disorder (44-46), obsessive-compulsive disorder (47) and insomnia (48) in both guided and unguided self-help programs. Specifically, a review of 30 studies found that CBT-based self-help interventions significantly reduced both anxiety and depression. Studies also show that people tend to maintain their progress over time, which is very encouraging (49). Furthermore, since patients can return to the program at their convenience to access treatment information, this may facilitate learning and retention

Despite a growing interest in the field of web-based psychological interventions, Internet-based CBT selfhelp programs remain underdeveloped. 
To fill this important gap in the literature and to properly meet the need of the population, it is crucial to promote and investigate the impact of this Internet-based psychological intervention in increasing individuals' emotional well-being.

For this reason, the present RCT aims to explore the effectiveness of a novel Internet-based CBT self-help program specifically developed to address the immediate stress and prevent long-term psychological consequences of the COVID-19 pandemic among Italians both living in the county and abroad. To assess its impact, the effects of the RinasciMENTE program will be compared with a waiting list (WL) condition on selected self-reported measures immediately following the 8-week intervention and at 6- and 12-month follow-up.

The primary hypothesis that will be tested is that the program will be feasible and effective in increasing the psychological functioning of the person.

The secondary hypothesis will test whether participants assigned to the experimental condition will show a reduced level of stress, anxiety, depression, and fear of COVID-19, as well as improved emotion regulation strategies, self-efficacy, and psychological well-being than those in the WL condition at treatment termination.

Moreover, participants in the experimental condition will be expected to present maintained outcomes or further decreased psychological symptoms at 6 and 12-month after the end of the treatment program.

\section{Methods}

\section{Design}

The project consists of a prospective, randomized, open, and parallel group-controlled study with two arms: an experimental arm with 8 online weekly CBT self-help sessions, and a waiting list (WL) control arm.

\section{Ethical Statement}

The study was approved by the Ethical Committee of the Catholic University of Milan, Italy (ID: 25-21). All procedures performed in the study will be run following the ethical standards of the institutional and/or national research committee and with the Helsinki Declaration and its later amendments or comparable ethical standards.

\section{Patients and public involvement}

Participants from the general population will be recruited through advertisements placed on social media platforms (i.e., Facebook, Instagram, Twitter), and online webinars on the topic.

The recruitment materials will include details about the study aim, the treatment delivered, and the conditions for participation, together with the weblink to access the program. 
Inclusion criteria for the participants into the study will be: (A) being fluent in the Italian language; (B) being over 18 years old; (C) providing online informed consent; and (D) showing mild/subthreshold levels of symptoms at the 12-Item General Health Questionnaire (GHQ-12) - Italian version (50, 51) - the most extensively used screening tool assessing the severity of common mental disorders over the past few weeks using a 4-point Likert-type scale (from 0 to 3 ). Its total score ranges from 0 to 36 , with high scores indicating worse health status. Only participants who score $\geq 15$ on the GHQ and show moderate levels of symptoms at the clinical interview will be included in the study.

Participants will be excluded from the program if: (A) presenting visual, hearing, or cognitive impairments that will prevent them from receiving and following the intervention; (B) suffering from severe psychiatric disorders according to the Diagnostic and Statistical Manual of Mental Disorders (DSM 5) (52); (C) lacking basic computer skills or internet access.

Respondents will not be excluded if already receiving psychopharmacological therapy or psychologicalpsychotherapeutic support.

\section{Sample size calculation}

The minimum sample size required to conduct this study was calculated using an a priori sample size calculator (G*Power 3.1.9.2 software) for $F$ tests (53-55). Participants were randomly divided into two groups: (A) iCBT self-help and (B) waiting list control. Moreover, participants will be measured in 3 moments: (1) before the intervention, (2) at the end of intervention (two months later), and (3) after 12 months from treatment termination. Due to the novelty of the study from which to derive realistic estimates of effect sizes, the partial $\eta 2$ was set a priori to assume a value of 0.02 - small effect size (56, 57 ) - which provides a Cohen's $f$ value of 0.143 . Moreover, the type I error (a) rate was set at 0.05 (twosided) and the Power $(1-\beta)$ was set at 0.95 , and the a priori correlation between repeated measures was set at 0.50 , according to general guidelines (56). Lastly, the non-sphericity correction was set to 1 . Results showed that there is a $95 \%$ chance of correctly rejecting the null hypothesis of no significant effect of the interaction with 128 participants in total (64 for each group).

\section{Randomization and blinding}

The random randomization scheme will be generated using the Web site Randomization.com (58). Allocation concealment will be ensured by the program generating an anonymous code for each participant that will be associated with the randomization sequence. Due to the nature of the intervention, the treatment group allocation cannot be concealed from the participants, nor the research team and assessor of outcomes. Participants will be assigned to one of two conditions within 2 working days from their baseline assessment (Figure 1).

\section{Measures}


Demographic and clinical information. Information about age, gender, education, civil status, weight, and high - used to calculate the Body Mass Index $\left(\mathrm{BMl}, \mathrm{kg} / \mathrm{m}^{2}\right)$ in presence of dysfunctional eating behaviors will be self-reported online at baseline. Subjects will be also asked to provide information about the country where they are currently living, their work situation (before and after the COVID-19 pandemic), and the personal experience they made of both COVID-19 pandemic and related preventive measures.

The Italian version of the following psychological measures will be also collected at baseline (T0), treatment termination after 2 months (T1), at 6-month (T2), and 12-month (T3) follow-up in the experimental condition only). Please see Figure 2 for a detailed project timeline.

\section{Primary outcome:}

Outcome Questionnaire (OQ-45) (59) composed by 45 items rated on a 5-point Likert scale ( $0=$ Never -4 = Almost always) assessing the treatment progresses across 3 different domains: Symptom Distress (SD - 25 items: $2,3,5,6,8,9,10,11,13,15,22,23,24,25,27,29,31,33,34,35,36,40,41,42$ and 45), Interpersonal Relations (IR - 11 items: 1, 7, 16, 17, 18, 19, 20, 26, 30, 37 and 43), and Social Role (SR - 9 items: $4,12,14,21,28,32,38,39$ and 44 ). The $0 Q-45$ total score is calculated by summing the score of the three subscales. Total score (range 0 to 180) is calculated by the sum of the items.

\section{Secondary outcomes:}

Perceived stress scale (PSS) (60), composed of 10 items rated on a 5-point Likert scale (from $0=$ never to 4 = very often) assessing the degree to which situations in one's life are appraised as stressful. PSS scores are obtained by reversing responses (i.e., $0=4,1=3,2=2,3=1$ and $4=0$ ) to the four positively stated items (items $4,5,7$, and 8 ) and then summing across all scale items. The total score (range 0 to $40)$ is calculated by the sum of the items.

Emotional regulation questionnaire (ERQ) (61), composed of 10 items rated on a 7-point Likert scale (from 1 = strongly disagree to 7 = strongly agree) assessing respondents' tendency to regulate their emotions in two ways: Cognitive Reappraisal (items 1, 3, 5, 7, 8, and 10) and Expressive Suppression (items $2,4,6$, and 9 ). The total score (range 7 to 70 ) is calculated by the sum of the items.

Depression Anxiety Stress Scales-Short Version (DASS-21) (62), composed by 21 statements rated on a 4point Likert scale (from $0=$ did not apply to me at all to $3=$ applied to me very much) assessing the negative emotional states of depression, anxiety, and stress. Each of the three DASS-21 scales contains 7 items: Depression: dysphoria, hopelessness, devaluation of life, self-deprecation, lack of interest/involvement, anhedonia, and inertia - (Items 3, 5, 10, 13, 16, 17, and 21); Anxiety. autonomic arousal, skeletal muscle effects, situational anxiety, and subjective experience of anxious affect - (Items $2,4,7,9,15,19$, and 20); Stress: levels of chronic nonspecific arousal, difficulty relaxing, nervous arousal, and being easily upset/agitated, irritable/over-reactive and impatient - (Items 1, 6, 8, 11, 12, 14, and 18). Sum scores are computed by adding up the scores on the items per (sub)scale and multiplying them by 2. Sum scores for the total DASS-total scale thus range between 0 and 126, and those for each of the 
subscales may range between 0 and 42 . Ranges of scores correspond to levels of symptoms, ranging from "normal" to "extremely serious". Notably, the DASS-21 has been recently used to investigate the impact of the COVID-19 pandemic on the physical and mental health of different populations worldwide (63-65).

Fear of COVID-19 Scale (FCV-19S) (66) composed by 7-item rated on a 5-point scale, ranging from 1 (from 1 = strongly disagree to 5 = strongly agree) reflecting cognitive, emotional, behavioral, and physiological manifestations of fear related to COVID-19. The total score (range 7 to 35 ) is calculated by the sum of the items. All items are positively worded, implying that higher scores indicate greater levels of fear.

General Self-efficacy Scale(GSES) (67), composed of 10 items rated on a 4-point Likert scale (from 1 = Not at all true to 4 = exactly true) assessing the individuals' confidence in their ability to cope with a variety of difficult or stressing situations. The total score is calculated by finding the sum of all items. For the GSE, the total score ranges between 10 and 40, with a higher score indicating more self-efficacy.

World Health Organization Quality of Life (WHOQOL)-BREF (68), composed of 26 items rated on a 5-point Likert scale assessing four domains: physical health (7 items on mobility, daily activities, functional capacity, energy, pain, and sleep), psychological health (6 items on self-image, negative thoughts, positive attitudes, self-esteem, mentality, learning ability, memory concentration, religion, and the mental status), social relationships (3 items on personal relationships, social support, and sex life), and environmental health (8 items related to financial resources, safety, health, and social services); it also contains QOL and general health items. The scores (range 25 to 130) are transformed linearly to a 0-100-scale.

Moreover, adherence will be measured through indicators such as the percentage of visited pages or the number of accesses.

Acceptability will be assessed by registering the participants' attrition rates and - for the experimental group only - by the use of the System Usability Scale (SUS)(69) composed of 10 statements scored on a 5-point Likert scale (from 1 = strongly disagree to 5 = strongly agree). The final score range from 0 to 100 , with a score above 68 indicating the absence of usability problems.

\section{Procedure}

Those interested in taking part in the program will be directed to the RinasciMENTE website (https://www.iterapi.se/sites/rinascimente/login) where they will find the written (online) consent, and after the acceptance of this, to each participant will be sent a document with information on the aims and requirements of the study.

They will be first asked to complete the GHQ-12 self-report questionnaires. Then, during the following 5 days, they will choose a time slot to be contacted for a clinical semi-structured interview lasting about 45 minutes, led by a certified clinical psychotherapist not involved in the study who will further assess the 
individual's eligibility to take part in the study. Additional information about the study and the randomization procedure will be also given to all respondents.

Next, the inclusion of each participant in the study and modules comprising the program will be discussed in a group by the professional who will conduct the interview and the research investigators (GP and VB), and the decision will be e-mailed to each participant within a week. Reasons for exclusion will be explained to the persons and - in presence of severe psychological disturbance - individuals will be suggested to ask for professional help.

Eligible subjects will be then randomly assigned to either the experimental (iCBT self-help) or control group (WL), and will receive an e-mail with a username and a customized link to create their password allowing them to log into the iterapi platform $(70,71)$. The iterapi is a safe platform with demonstrated efficacy in reducing symptoms of a wide range of disorders including somatic problems - such as hearing loss (72) and tinnitus (73) - to more traditional forms of psychological suffering, such as anxiety (74) and depression (75).

Participants in the experimental group will be required to make their first access to the platform and sign an electronic informed consent form within 24 hours, while those in the control condition will be able to access the online treatment once the experimental group will conclude the intervention (after two months).

The platform will be used for communication between the mental health professionals and the participants, for the delivery of the intervention, and the quantitative assessments.

Before entering the program, participants in the study will have to complete the self-report questionnaires assessing the above selected primary and secondary outcomes (TO).

The treatment will consist of 8 weekly modules, for a total duration of 2 months. Seven modules (Introduction, Behavioral activation, Dysfunctional beliefs, Acceptance, Stress management, Problem resolution and Plan of completion and maintenance) will be maintained for all participants, while one module will be selected tailored on the specific psychological needs of each participant. between the following: (1) Emotional school; (2) Anxiety and exposition; (3) Constant anxiety, (4) Social anxiety, (5) Panic attack, (6) Sleep quality, (7) Perfectionism; (8) Relaxation; (9) Management of difficult memories. The description of each module is reported in Table 1.

For the entire duration of the intervention, every Thursday participants will receive an e-mail communicating the update of the new material on the platform. Participants who will not access the material or that will not complete the suggested tasks and exercises will receive a weekly reminder containing a brief encouraging message every Monday.

At the end of the program (after two months - T1), participants in both conditions will be asked to fill in the baseline questionnaires again. Then, the experimental group will be also assessed after 6 - and 12 months from the term of the treatment (T2 and T3) (Figure 1). 


\section{Statistical analysis}

Statistical analyses were performed using SPSS software ver. 24.0 (76).

First, preliminary analyses will be performed to test the assumptions of both univariate and multivariate normality: if (strong) violations will be detected, robust methods or data transformation will be applied. Dropouts will be excluded from the study.

A missing values analysis will be run to see if the values are Missing Completely at Random (MCAR), or if there is some pattern among missing data. If there are no patterns detected, then pairwise or listwise deletion will be done to deal with missing data. However, if the missing values analysis detects a pattern, then imputation will be done.

The demographic characteristics will be reported as means and standard deviations for continuous variables, and frequencies and percentages for categorical variables.

The chi-square statistic will be used to test the association between treatment groups and sociodemographic and variables (i.e., age, gender, education, civil status, job), and correlation analysis will be used to test the association between quantitative variables.

The outcomes will be compared in the two treatment conditions using an Intention-To-Treat (ITT) approach. The significance level will be set at $5 \%$.

A repeated-measures ANOVA will be used to determine whether there are any between and within groups differences in the selected outcomes from baseline to treatment termination., Then repeated-measures within group ANOVA will test outcome differences over time baseline, end of treatment, 6- and 12-month follow-up) in the experimental group only (. Corrected effect sizes (Cohen's $d$ ) and significance at 95\% confidence interval $(95 \% \mathrm{Cl})$ will be calculated for both between-group and within-group differences, while for the total effect will be calculated the Cohen's $f$.

\section{Discussion}

To help and mitigate the impact of the COVID-19 pandemic on selected psychological parameters, the objective of this study is to evaluate the effectiveness of an Internet-based self-help intervention based on CBT principles and techniques, in reducing the level of stress, anxiety, depression, and fear of COVID-19, while increasing emotions self-regulation and perceived self-efficacy of Italians both living in the country and abroad. .

The online modality was chosen due to the current multiple barriers for face-to-face psychological interventions (i.e., mobility), and its largely acknowledged advantages, including greater flexibility or the possibility to reach rural or low-income population (77), and Italians living outside the country who look for psychological support in their language. Also, the self-help approach to therapy will be employed to 
further improve access to treatments and empower users to strengthen their self-management ability, while putting less strain on therapeutic resources (78) than conventional treatments do.

\section{Expected results}

The results of this RCT will provide evidence for the feasibility and effectiveness of the RinasciMENTE program in supporting the emotional well-being of the persons. Specifically, people are expected to show reduced levels of stress, anxiety, depression, and fear of COVID-19, as well as improved emotion regulation strategies, and self-efficacy. They are also expected to acquire the skills necessary to deal with the psychological consequences of social isolation during and after the COVID-19 outbreak.

Still, among the weaknesses of this study, it must be anticipated possible lack of internet access and digital literacy skills of some people.

Non-digital natives (i.e., older people) might, indeed, experience difficulties in the use of digital tools, and social isolation can pose additional challenges to their usage (i.e., chaotic home environments, limited privacy, or unreliable internet connection) (79).

The use of online intervention might also prevent effective controlling of confounding variables (i.e. environmental factors) that might impact treatment outcomes (80).

The results from this study will help to detect and address any usability problem, as Internet-based interventions, due to their characteristics and format, might be a suitable and feasible solution to mitigate the psychological impact of the virus outbreak and its related containment measures on the mental health status of the general population across the lifespan. Moreover, the findings will contribute to the further adaption of the self-help CBT programs.

In line with previous research findings, it is possible to predict that dropout rates may be higher with webbased interventions than in traditional face-to-face therapy (80), especially when self-help programs are used. To overcome this problem, participants in the study will receive a weekly reminder and motivational messages to access the online material. A positive relationship between each respondent and their referral therapist will be also supported, and while on the one hand, the professional remains available in case of need, the subject takes responsibility over the course of the treatment. The learning process is emphasized, so to help the person improve self-management skills and strategies to deal with their emotional problems.

Furthermore, the online format brings with it the advantage of allowing us to also support the population of Italians abroad, of which the literature does not report any in-depth analysis. RinascMENTE, therefore, represents the first attempt to provide online psychological support to the segment of the Italian population abroad.

\section{Conclusion}


Since the beginning of the COVID-19 pandemic, studies demonstrated a significant clinical and statistically decrease in mental health across populations worldwide. This burden is expected to continue in the aftermath making high demand for timely and pragmatic psychological interventions tailored to the unique and immediate needs of the general public. Preventive measures, fear of being infected, and financial losses give a reason for the implementation and use of digital self-help programs based on CBT principles and techniques - which might offer a range of self-management strategies to cope with emotional difficulties, besides reducing the cost of the health care system (81).

\section{Declarations}

\section{Ethics approval and consent to participate}

The study protocol and informed consent to participate were approved by the Research Ethics Committee of the Catholic University of Milan, Italy (25-21). All participants will sign in the online informed consent to participate before entering the study.

\section{Consent for publication}

Participants will give their consent for the anonymous publication of their data by signing the online informed consent form and information sheets

\section{Availability of data and materials}

All data will be identified only by a code, with personal details kept in a secure online platform with access only by the immediate research team.

\section{Competing interests}

The authors declare that they have no competing interests.

\section{Funding}

The author(s) received no financial support for the research, authorship, and/or publication of this article.

\section{Authors' contributions}

Conceptualization, V.B, M.S., and G.P.; methodology, G.P., and G.A.; writing-original draft preparation, V.B., and M.S.; writing-review and editing, G.P., G.A., GMM, and G.C.; visualization and supervision, G.P. and G.A.; project administration, G.P., and E.M. All authors have read and agreed to the published version of the manuscript.

\section{Authors' contributions}

Not applicable 


\section{Trial status}

Protocol Version 01 of February 2022

Start of recruitment: $21 / 02 / 2022$

\section{References}

1. Bonaccorsi G, Pierri F, Cinelli M, Flori A, Galeazzi A, Porcelli F, et al. Economic and social consequences of human mobility restrictions under COVID-19. Proceedings of the National Academy of Sciences. 2020;117(27):15530-5.

2. Pietrabissa G, Volpi C, Bottacchi M, Bertuzzi V, Guerrini Usubini A, Loffler-Stastka $H$, et al. The Impact of Social Isolation during the COVID-19 Pandemic on Physical and Mental Health: The Lived Experience of Adolescents with Obesity and Their Caregivers. International journal of environmental research and public health. 2021;18(6).

3. Xiong J, Lipsitz O, Nasri F, Lui LMW, Gill H, Phan L, et al. Impact of COVID-19 pandemic on mental health in the general population: A systematic review. Journal of Affected Disorders. 2020;277:55-64.

4. Nguyen HC, Nguyen MH, Do BN, Tran CQ, Nguyen TTP, Pham KM, et al. People with Suspected COVID-19 Symptoms Were More Likely Depressed and Had Lower Health-Related Quality of Life: The Potential Benefit of Health Literacy. Journal of Clinical Medicine. 2020;9(4).

5. Rogers JP, Chesney E, Oliver D, Pollak TA, McGuire P, Fusar-Poli P, et al. Psychiatric and neuropsychiatric presentations associated with severe coronavirus infections: a systematic review and meta-analysis with comparison to the COVID-19 pandemic. The lancet Psychiatry. 2020;7(7):611-27.

6. Rajkumar RP. COVID-19 and mental health: A review of the existing literature. Asian Journal of Psychiatry. 2020;52:102066.

7. Parola A, Rossi A, Tessitore F, Troisi G, Mannarini S. Mental Health Through the COVID-19 Quarantine: A Growth Curve Analysis on Italian Young Adults. Frontiers in Psychology. 2020;11:567484.

8. Guerrini Usubini A, Cattivelli R, Varallo G, Castelnuovo G, Molinari E, Giusti EM, et al. The Relationship between Psychological Distress during the Second Wave Lockdown of COVID-19 and Emotional Eating in Italian Young Adults: The Mediating Role of Emotional Dysregulation. Journal of personalized medicine. 2021;11(6).

9. Loffler-Stastka H, Bednar K, Pleschberger I, Prevendar T, Pietrabissa G. How to Include Patients' Perspectives in the Study of the Mind: A Review of Studies on Depression. Front Psychol. 2021;12:651423.

10. Cao W, Fang Z, Hou G, Han M, Xu X, Dong J, et al. The psychological impact of the COVID-19 epidemic on college students in China. Psychiatry Research. 2020;287:112934. 
11. Pietrabissa G, Simpson SG. Psychological Consequences of Social Isolation During COVID-19 Outbreak. Frontiers in psychology. 2020;11:2201.

12. Giusti EM, Pedroli E, D'Aniello GE, Stramba Badiale C, Pietrabissa G, Manna C, et al. The Psychological Impact of the COVID-19 Outbreak on Health Professionals: A Cross-Sectional Study. Frontiers in psychology. 2020;11:1684.

13. Ministero della Salute. Covid-19 - Situazione nel mondo. 2021.

14. Bertuzzi V, Semonella M, Bruno D, Manna C, Edbrook-Childs J, Giusti EM, et al. Psychological Support Interventions for Healthcare Providers and Informal Caregivers during the COVID-19 Pandemic: A Systematic Review of the Literature. International Journal of Environmental Research and Public Health. 2021;18(13).

15. Rossi Ferrario S, Panzeri A, Cerutti P, Sacco D. The Psychological Experience and Intervention in PostAcute COVID-19 Inpatients. Neuropsychiatric disease and treatment. 2021;17:413-22.

16. Pietrabissa G, Manzoni GM, Algeri D, Mazzucchelli L, Carella A, Pagnini F, et al. Facebook Use as Access Facilitator for Consulting Psychology. Australian Psychologist. 2015;40(4):299-303.

17. Rossi AA, Marconi M, Taccini F, Verusio C, Mannarini S. From Fear to Hopelessness: The Buffering Effect of Patient-Centered Communication in a Sample of Oncological Patients during COVID-19. Behav Sci (Basel). 2021;11(6).

18. Panzeri A, Rossi Ferrario S, Cerutti P. Psychological Differences Among Healthcare Workers of a Rehabilitation Institute During the COVID-19 Pandemic: A Two-Step Study. Frontiers in Psychology. 2021;12.

19. Maunder RG. Was SARS a mental health catastrophe? Gen Hosp Psychiatry. 2009;31(4):316-7.

20. Schwartz R, Sinskey JL, Anand U, Margolis RD. Addressing Postpandemic Clinician Mental Health : A Narrative Review and Conceptual Framework. Annals of internal medicine. 2020;173(12):981-8.

21. Panzeri A, Rossi Ferrario S, editors. Supporting rehabilitation patients with COVID-19 during the pandemic: Experiences from a technology-based psychological approach. CEUR Workshop Proceedings: Second Symposium on Psychology-Based Technologies - Psychobit, 2730; 2020.

22. Murphy R, Calugi S, Cooper Z, Dalle Grave R. Challenges and opportunities for enhanced cognitive behaviour therapy (CBT-E) in light of COVID-19. The Cognitive Behaviour Therapist. 2020;13.

23. Jassi A, Shahriyarmolki K, Taylor T, Peile L, Challacombe F, Clark B, et al. OCD and COVID-19: a new frontier. The Cognitive Behaviour Therapist. 2020;13.

24. Murray H, Grey N, Wild J, Warnock-Parkes E, Kerr A, Clark DM, et al. Cognitive therapy for posttraumatic stress disorder following critical illness and intensive care unit admission. The Cognitive Behaviour Therapist. 2020;13.

25. Wang C, Pan R, Wan X, Tan Y, Xu L, Ho CS, et al. Immediate Psychological Responses and Associated Factors during the Initial Stage of the 2019 Coronavirus Disease (COVID-19) Epidemic among the General Population in China. International journal of environmental research and public health. 2020;17(5). 
26. Kang L, Li Y, Hu S, Chen M, Yang C, Yang BX, et al. The mental health of medical workers in Wuhan, China dealing with the 2019 novel coronavirus. Lancet Psychiatry. 2020;7(3).

27. Shangguan F, Quan X, Qian W, Zhou C, Zhang C, Zhang XY, et al. Prevalence and correlates of somatization in anxious individuals in a Chinese online crisis intervention during COVID-19 epidemic. Journal of Affective Disorders. 2020;277:436-42.

28. Brog NA, Hegy JK, Berger T, Znoj H. An internet-based self-help intervention for people with psychological distress due to COVID-19: study protocol for a randomized controlled trial. Trials. 2021;22(1):171.

29. Wei N, Huang BC, Lu SJ, Hu JB, Zhou XY, Hu CC, et al. Efficacy of internet-based integrated intervention on depression and anxiety symptoms in patients with COVID-19. Journal of Zhejiang University Science B. 2020;21(5):400-4.

30. Pizzoli SMF, Marzorati C, Mazzoni D, Pravettoni G. An Internet-Based Intervention to Alleviate Stress During Social Isolation With Guided Relaxation and Meditation: Protocol for a Randomized Controlled Trial. JMIR research protocols. 2020;9(6):e19236.

31. Barak A, Hen L, Boniel-Nissim M, Shapira N. A Comprehensive Review and a Meta-Analysis of the Effectiveness of Internet-Based Psychotherapeutic Interventions. Journal of Technology in Human Services. 2008;26(2-4):109-60.

32. Griffiths KM, Farrer $L$, Christensen $\mathrm{H}$. The efficacy of internet interventions for depression and anxiety disorders: a review of randomised controlled trials. Medical Journal of Australia. 2010;192(11).

33. Andersson G. Internet interventions: Past, present and future. Internet Interventions. 2018;12:181-8.

34. Liu S, Yang L, Zhang C, Xiang YT, Liu Z, Hu S, et al. Online mental health services in China during the COVID-19 outbreak. The lancet Psychiatry. 2020;7(4):e17-e8.

35. Castelnuovo G, Pietrabissa G, Manzoni GM, Sicurello F, Zoppis I, Molinari E. Fighting the COVID-19 pandemic using the technology-based second-line in Italy and Lombardy: The urgent need of homebased remote monitoring systems to avoid the collapse of the hospital-centred first line. Journal of global health. 2020;10(2):010371.

36. Simpson S, Richardson L, Pietrabissa G, Castelnuovo G, Reid C. Videotherapy and therapeutic alliance in the age of COVID-19. Clinical psychology \& psychotherapy. 2021;28(2):409-21.

37. Shafran R, Clark DM, Fairburn CG, Arntz A, Barlow DH, Ehlers A, et al. Mind the gap: Improving the dissemination of CBT. Behaviour research and therapy. 2009;47(11):902-9.

38. Nobili RM, Gambazza S, Spada MS, Tutino AL, Bulfamante AM, Mariani A, et al. Remote support by multidisciplinary teams: A crucial means to cope with the psychological impact of the SARS-COV-2 pandemic on patients with cystic fibrosis and inflammatory bowel disease in Lombardia. International journal of clinical practice. 2021;75(7):e14220.

39. Wang H, Zhao Q, Mu W, Rodriguez M, Qian M, Berger T. The Effect of Shame on Patients With Social Anxiety Disorder in Internet-Based Cognitive Behavioral Therapy: Randomized Controlled Trial. JMIR mental health. 2020;7(7):e15797. 
40. Nordgreen T, Havik OE, Ost LG, Furmark T, Carlbring P, Andersson G. Outcome predictors in guided and unguided self-help for social anxiety disorder. Behaviour research and therapy. 2012;50(1):13-21.

41. Andersson G, Paxling B, Roch-Norlund P, Ostman G, Norgren A, Almlov J, et al. Internet-based psychodynamic versus cognitive behavioral guided self-help for generalized anxiety disorder: a randomized controlled trial. Psychotherapy and Psychosomatics. 2012;81(6):344-55.

42. Van't Hof E, Cuijpers P, Stein DJ. Self-help and Internet-guided interventions in depression and anxiety disorders: a systematic review of meta-analyses. CNS spectrums. 2009;14(2 Suppl 3):34-40.

43. Ciuca AM, Berger T, Crisan LG, Miclea M. Internet-based treatment for panic disorder: A three-arm randomized controlled trial comparing guided (via real-time video sessions) with unguided self-help treatment and a waitlist control. PAXPD study results. Journal of Anxiety Disorders. 2018;56:43-55.

44. Holst A, Nejati S, Bjorkelund C, Eriksson MC, Hange D, Kivi M, et al. Patients' experiences of a computerised self-help program for treating depression - a qualitative study of Internet mediated cognitive behavioural therapy in primary care. Scandinavian Journal of Primary Health Care. 2017;35(1):46-53.

45. Reins JA, Boss L, Lehr D, Berking M, Ebert DD. The more I got, the less I need? Efficacy of Internetbased guided self-help compared to online psychoeducation for major depressive disorder. Journal of Affective Disorders. 2019;246:695-705.

46. Vernmark K, Lenndin J, Bjarehed J, Carlsson M, Karlsson J, Oberg J, et al. Internet administered guided self-help versus individualized e-mail therapy: A randomized trial of two versions of CBT for major depression. Behaviour research and therapy. 2010;48(5):368-76.

47. Andersson E, Enander J, Andren P, Hedman E, Ljotsson B, Hursti T, et al. Internet-based cognitive behaviour therapy for obsessive-compulsive disorder: a randomized controlled trial. Psychological Medicine. 2012;42(10):2193-203.

48. Ye YY, Chen NK, Chen J, Liu J, Lin L, Liu YZ, et al. Internet-based cognitive-behavioural therapy for insomnia (ICBT-i): a meta-analysis of randomised controlled trials. BMJ Open. 2016;6(11):e010707.

49. Coull G, Morris PG. The clinical effectiveness of CBT-based guided self-help interventions for anxiety and depressive disorders: a systematic review. Psychological medicine. 2011;41(11):2239-52.

50. Balestrieri M, Carta MG, Leonetti S, Sebastiani G, Starace F, Bellantuono C. Recognition of depression and appropriateness of antidepressant treatment in Italian primary care. Social Psychiatry and Psychiatric Epidemiology. 2004;39(3):171-6.

51. Goldberg DP. The Detection of Psychiatric Illness by Questionnaire: Oxford University Press: Oxford.; 1972.

52. American Psychiatric Association. Diagnostic and statistical manual of mental disorders (5th ed.). Washington, DC2013.

53. Faul F, Erdfelder E, Buchner A, Lang AG. Statistical power analyses using G*Power 3.1: Tests for correlation and regression analyses. Behavior Research Methods. 2009;41(4):1149-60.

54. Pietrabissa G, Castelnuovo G, Jackson JB, Rossi A, Manzoni GM, Gibson P. Brief Strategic Therapy for Bulimia Nervosa and Binge Eating Disorder: A Clinical and Research Protocol. Front Psychol. 
2019;10:373.

55. Cattivelli R, Castelnuovo G, Musetti A, Varallo G, Spatola CAM, Riboni FV, et al. ACTonHEALTH study protocol: promoting psychological flexibility with activity tracker and mHealth tools to foster healthful lifestyle for obesity and other chronic health conditions. Trials. 2018;19(1):659.

56. Cohen J. Statistical power analysis for the behavioral sciences. press A, editor. London2013.

57. Eid M, Gollwitzer M, Schmitt M. Statistik und forschungsmethoden2017.

58. Castelnuovo G, Manzoni GM, Villa V, Cesa GL, Pietrabissa G, Molinari E. The STRATOB study: design of a randomized controlled clinical trial of Cognitive Behavioral Therapy and Brief Strategic Therapy with telecare in patients with obesity and binge-eating disorder referred to residential nutritional rehabilitation. Trials. 2011;12:114.

59. Chiappelli M, Lo Coco G, Gullo S, Bensi L, Prestano C. The Outcome Questionnaire 45.2. Italian validation of an instrument for the assessment of psychological treatments. Epidemiologia e Psichiatria Sociale. 2008;17(2):152-61.

60. Mondo M, Sechi C, Cabras C. Psychometric evaluation of three versions of the Italian Perceived Stress Scale. Current Psychology. 2019:1-9.

61. Balzarotti S, John OP, Gross JJ. An Italian Adaptation of the Emotion Regulation Questionnaire. European Journal of Psychological Assessment. 2010;26(1):61-7.

62. Bottesi G, Ghisi M, Altoè G, Conforti E, Melli G, Sica C. The Italian version of the Depression Anxiety Stress Scales-21: Factor structure and psychometric properties on community and clinical samples. Comprehensive Psychiatry. 2015;60:170-81.

63. Wang C, Tee M, Roy AE, Fardin MA, Srichokchatchawan W, Habib HA, et al. The impact of COVID-19 pandemic on physical and mental health of Asians: A study of seven middle-income countries in Asia. PloS one. 2021;16(2):e0246824.

64. Wang C, Tripp C, Sears SF, Xu L, Tan Y, Zhou D, et al. The impact of the COVID-19 pandemic on physical and mental health in the two largest economies in the world: a comparison between the United States and China. Journal of behavioral medicine. 2021;44(6):741-59.

65. Wang C, Lopez-Nunez MI, Pan R, Wan X, Tan Y, Xu L, et al. The Impact of the COVID-19 Pandemic on Physical and Mental Health in China and Spain: Cross-sectional Study. JMIR formative research. 2021;5(5):e27818.

66. Soraci P, Ferrari A, Abbiati FA, Del Fante E, De Pace R, Urso A, et al. Validation and Psychometric Evaluation of the Italian Version of the Fear of COVID-19 Scale. Int J Ment Health Addict. 2020:1-10.

67. Sibilia L, Schwarzer R, Jerusalem M. talian Adaptation of the General Self-Efficacy Scale: SelfEfficacy Generalizzata 1995 [Available from: http://userpage.fu-berlin.de/ health/italian.htm.

68. De Girolamo G, Rucci P, Scocco P, Becchi A, Coppa F, D'Addario A, et al. Quality of life assessment: validation of the Italian version of the WHOQOL-Brief. Epidemiologia e Psichiatria Sociale. 2000;9(1):45-55. 
69. Brooke J. SUS: A "quick and dirty" usability scale. In: Jordan PW, Thomas B, Weerdmeester BA, McClelland IL, editors. Usability evaluation in industry. London: Taylor \& Francis; 1996. p. 189-94.

70. Vlaescu G, Alasjo A, Miloff A, Carlbring P, Andersson G. Features and functionality of the Iterapi platform for internet-based psychological treatment. Internet Interventions. 2016;6:107-14.

71. Semonella M, Vilchinsky N, Dakel R, Biliunaite I, Pietrabissa G, Andersson G, editors. SOSteniamoci: An internet-based intervention to support informal caregivers. PSYCHOBIT; 2020.

72. Thoren ES, Oberg M, Wanstrom G, Andersson G, Lunner T. A randomized controlled trial evaluating the effects of online rehabilitative intervention for adult hearing-aid users. International journal of audiology. 2014;53(7):452-61.

73. Hesser H, Gustafsson T, Lunden C, Henrikson O, Fattahi K, Johnsson E, et al. A randomized controlled trial of Internet-delivered cognitive behavior therapy and acceptance and commitment therapy in the treatment of tinnitus. Journal of consulting and clinical psychology. 2012;80(4):649-61.

74. Tulbure BT, Szentagotai A, David O, Stefan S, Mansson KN, David D, et al. Internet-delivered cognitivebehavioral therapy for social anxiety disorder in Romania: a randomized controlled trial. PloS one. 2015;10(5):e0123997.

75. Johansson R, Sjoberg E, Sjogren M, Johnsson E, Carlbring P, Andersson T, et al. Tailored vs. standardized internet-based cognitive behavior therapy for depression and comorbid symptoms: a randomized controlled trial. PloS one. 2012;7(5):e36905.

76. IBM Corp. IBM SPSS Statistics for Windows, Version 24.0. In: Armonk NIC, editor. Released 2016.

77. Barak A, Grohol JM. Current and future trends in internet-supported mental health interventions. Journal of Technologies at Human Services. 2011;29:155-96.

78. Newman MG, Szkodny LE, Llera SJ, Przeworski A. A review of technology-assisted self-help and minimal contact therapies for anxiety and depression: is human contact necessary for therapeutic efficacy? Clinical psychology review. 2011;31(1):89-103.

79. Liverpool S, Mota CP, Sales CMD, Cus A, Carletto S, Hancheva C, et al. Engaging Children and Young People in Digital Mental Health Interventions: Systematic Review of Modes of Delivery, Facilitators, and Barriers. Journal of medical Internet research. 2020;22(6):e16317.

80. Botella C, Mira A, Herrero R, García-Palacios A, Baños R. Un programa de intervención auto-aplicado a través de Internet para el tratamiento de la depresión: "Sonreír es divertido". Aloma. 2015;33(2):3948.

81. Castelnuovo G, Pietrabissa G, Cattivelli R, Manzoni GM, Molinari E. Not Only Clinical Efficacy in Psychological Treatments: Clinical Psychology Must Promote Cost-Benefit, Cost-Effectiveness, and Cost-Utility Analysis. Front Psychol. 2016;7:563.

\section{Table}

Table 1 PDF is available in the Supplemental Files section. 
Figures

\section{Recruitment and Final Screening}

Randomization $(\mathrm{n}=128)$

\begin{tabular}{|c|}
\hline $\begin{array}{c}\text { Experimental Group } \\
\mathrm{n}=64\end{array}$ \\
\hline $\begin{array}{c}\text { Baseline } \\
\text { Assessment (T0) }\end{array}$ \\
\hline Internet-based Intervention \\
\hline Post-Treatment \\
Assessment (T1) \\
\hline Follow-up \\
6-month (T2) \\
\hline Follow-up \\
12-month (T3)
\end{tabular}

\section{Figure 1}

Flow chart of the RinasciMENTE study 


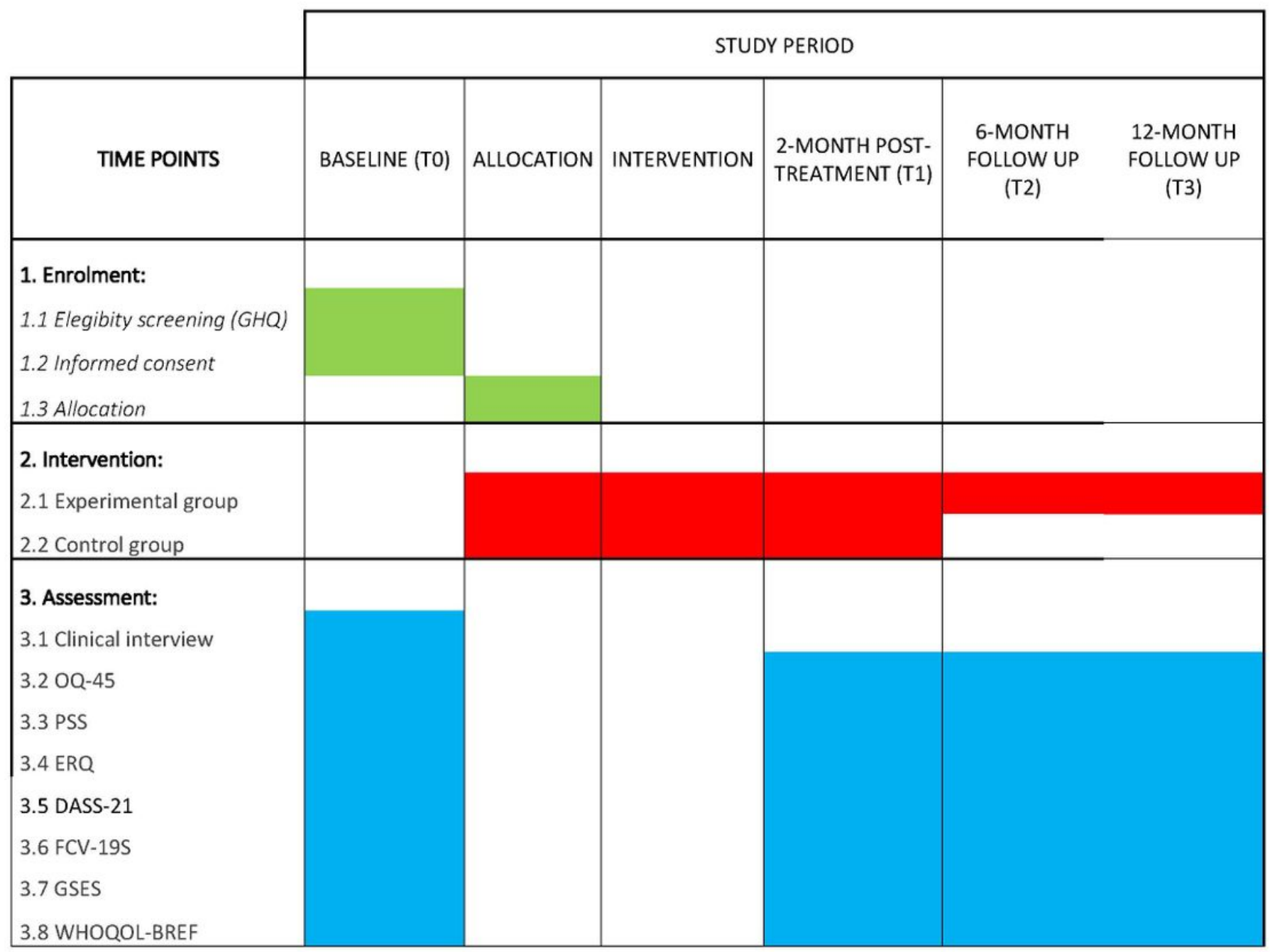

Figure 2

Gantt of the RinasciMENTE study

\section{Supplementary Files}

This is a list of supplementary files associated with this preprint. Click to download.

- SPIRITRinasciMENTEVBjan2022.doc

- Tabella1Moduli.pdf 